\title{
L’autonomie, illusion ou projet de société ?
}

\section{RÉSUMÉ}

Selon une analyse très répandue aujourd'hui, la société contemporaine serait caractérisée par une vaste autonomie des acteurs; pourtant, ce n'est pas parce que l'hétéronomie autoritaire a régressé au cours des dernières décennies que toute forme d'hétéronomie a disparu, ni que l'autonomie s'étend automatiquement à toute la société. Les domaines dans lesquels le terme " autonomie " est actuellement d'usage courant sont multiples et les acceptions scientifiques du concept fort diverses; est-ce à dire qu'un même mot est employé pour désigner des faits irréductibles entre eux ou bien n'est-il de véritable autonomie que complexe, tant individuelle que collective ? En tout cas, l'autonomie est moins, aujourd'hui, une réalité, qu'un projet de société, à la fois exigeant, limité et novateur.

Mots clés : Autonomie, Hétéronomie, Individu, Communauté, Société, Démocratie.

\section{SUMMARY}

It is often thought that contemporary society is characterized by its actors' great autonomy. However, this is not sure: firstly, it is not because authoritarian heteronomy has regressed during recent decades that all forms of heteronomy have disappeared, and, secondly, the regression of heteronomy does not mean an automatic extension of autonomy to all society. The fields in which the term " autonomy " is currently in everyday use are manifold, and the scientific meanings of the concept are extremely varied; does this mean that the same word is employed to signify completely different points or that true autonomy can only be complex, at both an individual and a collective level ? In any case, today, autonomy is less a reality than a project for society, at once demanding, limited and innovative.

Key words : Autonomy, Heteronomy, Individual, Community, Society, Democracy.

Le $x_{x \mid}{ }^{e}$ siècle semble marquer l'aboutissement d'une très longue quête d'autonomie. Si dans la Grèce antique, l'autonomia était la possibilité pour une cité ${ }^{1}$ de faire ses propres lois sans être sous l'emprise d'une tutelle étrangère, le terme d'autonomie a été ultérieurement étendu aux individus ${ }^{2}$. L'impulsion est venue de penseurs religieux qui mettaient l'accent sur le rôle de la conscience individuelle Thomas d'Aquin, notamment, puis Luther et Calvin - et des humanistes de la Renaissance. L'autonomie est ensuite devenue l'un des grands desseins de la philosophie des Lumières et du projet de modernité. Aujourd'hui, le mot « autonomie »

1. Le terme n'était appliqué qu'aux cités qui, se trouvant dans l'orbite d'un puissant voisin, voulaient préserver leur relative souveraineté ; il n'était pas employé à propos des États forts. (Ostwald, 1982).

2. L'idée d'autonomie individuelle, en revanche, était déjà au cœur de la pensée d'Aristote (Éthique à Nicomaque) et des Stoïciens. 
est très employé dans la vie quotidienne et le langage courant; et surtout, beaucoup de spécialistes de sciences humaines et sociales s'accordent à considérer que l'autonomie est devenue une dimension essentielle de la vie sociale contemporaine. Cet avis n'est cependant pas unanime et, dans l'un de ses derniers ouvrages (1997), Cornelius Castoriadis, théoricien engagé du "projet d'autonomie », écrivait que ce projet subit à présent « une éclipse prolongée. [...] Un nouveau type anthropologique d'individu émerge, défini par l'avidité, la frustration, le conformisme généralisé ${ }^{3}$ »... Tout dépend, certes, de l'idée que l'on se fait de l'autonomie; or, la diversité des définitions de ce concept est impressionnante. Gerald Dworkin en donne une dizaine, très disparates ${ }^{4}$.

Or, souligne Onora O'Neill dans un article récent, non seulement la liste de Dworkin est loin d'être complète, mais les rares caractéristiques constantes de l'autonomie qu'il a cru déceler ne le sont pas ${ }^{5}$. Encore demeurons-nous pour l'instant dans le domaine de la philosophie; mais le rayonnement du concept d'autonomie est bien plus vaste, explique Ruth Lapidoth :

« De nos jours, le terme est utilisé dans différents domaines : en philosophie et dans les disciplines dérivées (le droit pour un individu de vivre selon sa volonté) ; en sciences naturelles et en médecine (l'organe autonome fonctionne indépendamment des autres organes); en droit, en science politique et en relations internationales. Dans ce dernier groupe de disciplines - droit et science politique - on utilise le terme en lui donnant plusieurs connotations : pour certains, il désigne le droit d'une personne ou d'un organe public d'agir discrétionnairement, pour d'autres, il est synonyme d'indépendance. ॥" "Selon une troisième acception que nous avons préférée, il signifiera qu'une entité infra-étatique détient des pouvoirs - souvent exclusifs - de législation, d'administration et, dans certains cas aussi, un pouvoir judiciaire dans des sphères spécifiques. ${ }^{7}$

Face à cette profusion de sens, rappelons l'étymologie - non contestée - du mot autonomie ; il provient de deux mots grecs : auto, "soi-même ", et nomos, " loi » ou " règle ». Rappelons, surtout, la conception kantienne de l'autonomie, qui pourra servir de point de départ à notre réflexion. Selon Kant, l'autonomie est le choix qui ne repose que sur la volonté propre de l'homme, établissant une «législation universelle ». Tout choix qui serait soumis à un élément " extérieur » tel que l'attrait, l'intérêt, la contrainte, le devoir, la loi, la nécessité, etc., relèverait, au contraire, de l'hétéronomie; d'autre part, la législation propre de l'homme est universelle dans la mesure où il agit « selon la maxime qui peut en même temps s'ériger elle-même en loi universelle ${ }^{8}$. C'est-à-dire que, face à une situation donnée, un individu est autonome, selon Kant, si, réfléchissant à sa conduite, il choisit volontairement et librement de se comporter de la façon qu'il juge être universellement la meilleure. Dans tout autre cas (si, par exemple, il suit les ordres qu'il a reçus, s'il obéit à la loi, s'il se conforme à son désir, etc.), il se comporte de façon hétéronome.

\footnotetext{
. Castoriadis, 1997, p. 75.

${ }^{4}$. Dworkin, 1998, p. 6.

5. O'Neill, 2004, p. 2.

${ }^{6}$. Lapidoth, 1997, p. 29.

7. Lapidoth, in Le Coadic 2003, p. 266.

${ }^{8}$. Kant, 2000, p. 164, souligné par l'auteur.
} 
Compte tenu de la présence assez forte du concept d'autonomie tant dans le discours scientifique contemporain que dans le langage courant et, compte tenu de l'absence de consensus formel sur sa signification, nous nous poserons, en premier lieu, la question de savoir ce que peuvent être les réalités de l'autonomie dans la société contemporaine. Ce n'est qu'après avoir étudié les caractéristiques de notre société qui font qu'on parle aujourd'hui d'autonomie que nous chercherons à voir s'il existe une cohérence du concept malgré l'apparente confusion de départ. Nous pourrons alors nous poser la question de savoir si un projet sous-tend aujourd'hui la réalité — ou la quête — d'autonomie.

\section{Quelle réalité ?}

\section{A. Une apparence d'autonomie...}

La "révolution des mœurs " des années 1960 a profondément marqué les pays occidentaux de son empreinte. La diffusion massive de la pilule contraceptive, en particulier, a libéré les femmes du risque de procréation. Les mouvements sociaux (féministes, d'une part, " hippies ", de l'autre) ont contribué à une modification des rapports sociaux de sexe. Les femmes ont pu disposer plus librement de leur corps et la recherche du plaisir sexuel est devenue socialement acceptable. De plus, la tolérance envers l'homosexualité s'est accrue et plusieurs pays ont adopté une législation qui reconnaît les mêmes droits aux homosexuels qu'aux hétérosexuels. La liberté sexuelle apparaît ainsi désormais comme une dimension fondamentale de l' « autonomie » individuelle ${ }^{9}$.

D'autre part, la famille patriarcale, où « l'autorité dominante d'un homme adulte [...] s'exerce de façon stable sur l'ensemble du groupe familial ${ }^{10}$, est en crise dans tous les pays industrialisés. Les couples se forment de plus en plus tard et beaucoup ne se marient pas, l'âge moyen à la naissance du premier enfant est de plus en plus élevé, la part des naissances hors mariage s'est considérablement accrue depuis les années 1970, le taux de divorce se stabilise à un niveau très élevé, les remariages sont fréquents et conduisent souvent à la formation de familles recomposées ; il n'y a plus de modèle unique mais une pluralité de types familiaux entre lesquels les individus paraissent évoluer en toute "autonomie ": union libre, familles monoparentales, couples non-cohabitants, familles recomposées, couples homosexuels...

Par ailleurs, en Europe occidentale, la pratique religieuse s'est complètement effondrée au cours des dernières décennies et les Églises traditionnelles ont perdu beaucoup de leur ascendant sur la population. En revanche, de nouveaux mouvements religieux se développent aujourd'hui dans le monde et, surtout, le syncrétisme et la subjectivisation se répandent largement: de plus en plus, chacun définit lui-même sa propre religion, indépendamment des Églises. C'est le believing without belonging, "croire sans appartenir ${ }^{11}$ : les individus " bricolent " en toute « autonomie » et combinent les croyances de leur enfance avec des bribes d'autres religions ou croyances.

D'autre part, plusieurs vagues de revendications liées à l'ethnicité se sont succédé depuis la Seconde Guerre mondiale dans les pays industrialisés: les unes de

\footnotetext{
${ }^{9}$. Même si la réalité est nettement plus complexe (cf. Daoust, 2005).

${ }^{10}$. Castells, 1998, tome II, p. 174.

${ }^{11}$. Davie, 1994.
} 
tendance autonomiste, analysées en termes de "nouveaux mouvements sociaux " par Alain Touraine ${ }^{12}$, d'autres à caractère franchement nationaliste, et d'autres encore relevant d'une mise en forme ethnique de revendications sociales. Ces vagues successives ont contribué à alimenter des phénomènes d'identification collective et à créer des "foyers identitaires ${ }^{13}$ beaucoup plus larges que les milieux militants: nombreux sont, en effet, les individus qui choisissent de construire leur spécificité en revendiquant, de façon "autonome », une appartenance ethnique plus ou moins fondée mais souvent très enthousiaste.

Enfin, alors que la démocratie représentative s'étend largement à travers le monde, les populations des États démocratiques les plus anciens et les plus prospères perdent confiance en leurs élites politiques et réduisent leur participation électorale. Contrairement à ce qui est souvent avancé, cela ne signifie pas que ces populations se désintéressent de la politique mais simplement qu'elles ne se reconnaissent plus dans la vie politique traditionnelle. Peut-être parce qu'elle est complètement focalisée sur l'État-nation et que celui-ci, selon la formule de Daniel Bell, est devenu too small for the big problems of life, and too big for the small problems of life ${ }^{14}$ ? Beaucoup de citoyens préfèrent en tout cas rejoindre des associations, qui leur permettent de s'engager de façon «autonome », que de compter sur leurs représentants. Tout cela, néanmoins, relève-t-il vraiment de l'autonomie?

\section{B. ... dont la réalité peut être contestée}

Dans les domaines que nous venons d'examiner et peut-être dans beaucoup d'autres, les individus d'aujourd'hui paraissent beaucoup moins sous l'emprise de l'hétéronomie que ne l'étaient leurs pères: ils puisent nettement moins que ces derniers les principes de leur action dans les règles sociales. Mais peut-on pour autant parler d'autonomie, c'est-à-dire d'auto-production par les individus de leurs propres normes et de leurs principes d'action? Sans même se poser la question de l'universalité de la « législation » que l'homme devrait produire pour être pleinement autonome au sens kantien, doit-on déduire d'un apparent affaissement de l'hétéronomie que celle-ci laisse en quelque sorte automatiquement la place à l'autonomie? Ne peut-on pas envisager, au contraire, que l'hétéronomie cède au moins en partie la place à l'anomie, c'est-à-dire à l'absence de normes et de valeurs communes?

Certes, il n'est pas sûr que le recours à un concept aussi complexe que l'anomie vienne éclairer notre lanterne sur les ambiguïtés de l'autonomie ${ }^{15}$. L'idée que nous voudrions, néanmoins, introduire ici est que l'affaissement apparent de l'hétéronomie, s'il est vérifié, ne place pas nécessairement les acteurs sociaux en situation de produire leurs propres normes mais peut-être face à un vide déstabilisant. Cette notion de "vide " a été particulièrement mise en avant par Gilles Lipovetsky ${ }^{16}$ qui, cependant, ne l'associe pas à l'anomie mais à une «nouvelle signification de l'autonomie ${ }^{17}$. Alain Ehrenberg parle également d'autonomie : « L'autonomie devient

\footnotetext{
12 . Touraine et al., 1981.

13 . Gauchet, 1998.

14 . Bell, 1987, p. 14.

15. Cf. Besnard, 1987. En particulier p. 380-381 et 387-388.

${ }^{16}$. Lipovetski, 1983. Cf. notamment p. 108.

${ }^{17}$. Lipovetsky, 1983, p. 12.
} 
une contrainte de masse », écrit-il en effet $^{18}$, qui se traduit par une montée de la dépression ${ }^{19}$. Son analyse se place dans la continuité des travaux de Marcel Gauchet sur le désenchantement du monde ${ }^{20}$. Mais l'autonomie peut-elle être une contrainte ? et chacun a-t-il les moyens d'élaborer sa «propre législation »? La montée de la dépression dans le monde aujourd'hui ${ }^{21}$ révèle-t-elle vraiment l'autonomie des acteurs sociaux ou, au contraire, le sentiment que certains d'entre eux éprouvent d'être démunis, incapables d'être autonomes? Cette question que nous posons fait immanquablement écho aux travaux de Durkheim sur le suicide anomique ${ }^{22}$. Cependant, tous les individus qui, fort heureusement, ne dépriment ni ne se suicident — et qui paraissent donc échapper à l'anomie —, sont-ils réellement autonomes ?

Les comportements collectifs contemporains, écrivions-nous précédemment, semblent attester que les individus d'aujourd'hui sont moins sous l'emprise de I'hétéronomie que ne l'étaient leurs pères. Est-ce néanmoins exact? Une grande partie des comportements avérés ne relèveraient-ils pas plutôt de ce " conformisme généralisé " que Castoriadis mettait en cause ? Quel impact ont la publicité, les médias et l'industrie cinématographique sur la structuration des représentations sociales ? Ne contribuent-ils pas à la diffusion d'un modèle de société ? Ne sont-ils pas des agents producteurs d'hétéronomie (même s'il est bien malaisé de mesurer la portée réelle de leur influence ${ }^{23}$ ) ? En fait, l'hétéronomie paraît d'autant moins avoir disparu qu'à ces agents hétéronormeurs externes - si l'on peut dire -, il convient d'en ajouter d'autres, plus puissants encore. Le premier d'entre eux n'est-il pas notre inconscient ? Comme l'écrit Castoriadis, en effet: "Si à l'autonomie, la législation ou la régulation par soi-même, on oppose l'hétéronomie, la législation ou la régulation par un autre, l'autonomie, c'est ma loi, opposée à la régulation par l'inconscient qui est une loi autre, la loi d'un autre que moi. " ${ }^{24}$ En quoi les individus contemporains seraient-ils moins soumis à leur inconscient et à leurs pulsions que ne l'étaient leurs pères ? En quoi seraient-ils donc plus autonomes ? Enfin - et corrélativement, la famille est l'autre puissant producteur d'hétéronomie. Certes, la famille patriarcale est aujourd'hui en crise, avons-nous dit. Mais, pour autant, en quoi, pourrions-nous affirmer que les individus contemporains seraient moins profondément influencés par leur milieu familial que ne l'ont été leurs ascendants ? Là encore, en quoi seraient-ils vraiment plus autonomes?

\section{C. ... mais qui n'est pas dénuée de fondement}

Récapitulons. Beaucoup de comportements présentent aujourd'hui toutes les apparences de l'autonomie ; or, simultanément, on constate une progression du mal vivre et de la dépression. Est-ce à dire que l'autonomie - la production de leurs propres normes par les acteurs sociaux, en l'absence d'hétéronomie - provoque ce mal-être ? Ou, au contraire, est-ce l'anomie qui est en cause, c'est-à-dire l'absence de normes sociales claires et rassurantes, et l'embarras qui en résulte pour certains acteurs sociaux, déroutés par un univers non borné ? II n'est pas aisé de répondre à la question. Sans doute l'autonomie peut-elle être épuisante et déprimante.

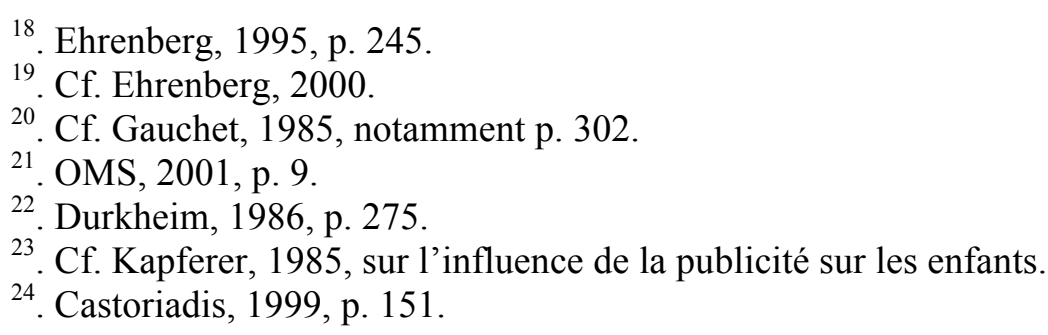


Rappelons-nous, à ce sujet, ce que Durkheim écrivait du "suicide égoïste » et, en particulier, le lien qu'il établissait entre l'autonomie de la conscience et le penchant au suicide des protestants ${ }^{25}$. D'un autre côté, l'anomie peut vraisemblablement être déconcertante et anxiogène. Or, à cet égard, I' " anomie conjugale " qu'évoquait Durkheim dans Le Suicide - caractérisée par le " mal de l'infini » du célibataire qui « aspire à tout et [que] rien ne [...] contente ${ }^{26}$ et I' " affaiblissement de la réglementation matrimoniale ${ }^{27}$ — n'est rien par rapport à la liberté de mœurs qui règne dans la plupart des sociétés occidentales contemporaines. II est donc possible que notre société soit caractérisée non par une généralisation de l'autonomie mais par la combinaison de deux phénomènes partiels, autonomie et anomie, qui pourraient, l'un comme l'autre, résulter, selon la terminologie de Durkheim, d'un " même état de désagrégation " ${ }^{28}$, soit, en d'autres termes, d'un affaiblissement de l'hétéronomie.

Est-ce si sûr, cependant ? Nous avons vu, en effet, que, d'une part, les principaux organes producteurs d'hétéronomie - l'inconscient et la famille - restent toujours très puissants et que, d'autre part, de nouveaux agents hétéronormeurs se sont développés (publicité, médias, etc.). En fait, ce qui distingue la situation contemporaine des sociétés occidentales sur le plan qui nous occupe n'est pas la disparition de l'hétéronomie, mais le fait que le pouvoir des institutions d'imposer I'hétéronomie de façon autoritaire décline : les Églises, les États, les partis politiques et les familles ne peuvent plus imposer sans discussion leur conception du monde et de la vie, ni assigner autoritairement sa place à chacun. Cet effondrement de I'hétéronomie autoritaire, cependant, ne suffit pas à garantir l'autonomie. D'une part, avons-nous constaté, quand l'hétéronomie semble disparaître, elle peut laisser la place aussi bien à de l'anomie qu'à de l'autonomie. D'autre part, l'effondrement de I'hétéronomie autoritaire se traduit, certes, par une perte d'influence des dogmes de tout ordre (religieux, politiques, sociaux ou culturels...), mais aussi par le maintien ou le développement d'une autre hétéronomie, profonde et insidieuse. Comment, en effet, prétendre vaincre ses pulsions inconscientes ou se dégager de ses influences familiales ? Comment échapper au modèle de société et aux valeurs que diffusent implicitement la publicité et certains médias? Et si on ne le fait pas, est-on autonome?

Car une seconde caractéristique de notre époque semble être la désirabilité de l'autonomie. Si elle est sans doute beaucoup moins répandue qu'on a pu l'affirmer, l'autonomie n'en est pas moins largement présentée aujourd'hui comme un objectif que chacun doit atteindre pour avancer sur la voie du bonheur... Une véritable injonction à l'autonomie se répand dans notre société (aussi bien dans le monde du travail $^{29}$ qu'à l'école $^{30}$, dans la vie quotidienne ${ }^{31}$, etc.), qui est totalement paradoxale puisque, par définition, l'autonomie ne peut pas être imposée mais relève de la « législation propre de l'homme » et de rien d'autre ${ }^{32}$.

${ }^{25}$. Durkheim, 1986, p. 157.

${ }^{26}$. Durkheim, 1986, p. 304.

27. Idem, p. 305.

${ }^{28}$. Idem, p. 440.

${ }^{29}$. Cf. Everaere, 2001.

30. Cf, entre autres, Freire, 2004.

31. Cf. Bouvier, 2005 et, sur un tout autre plan, parmi de nombreux ouvrages du même type, Filliozat, 1998.

32. Cf. Boltanski et Chiapello, 1999, sur l'injonction autoritaire au projet. 
En conclusion de cette première partie, il fait peu de doute que l'hétéronomie autoritaire s'effondre mais elle laisse la place à une réalité complexe où l'autonomie semble jouer les arlésiennes... Peut-être notre conception de l'autonomie, marquée par l'approche kantienne, est-elle trop exigeante et irréaliste ? II est temps, à présent, de réfléchir à la cohérence du concept, dans une perspective sociologique.

\section{Quelle cohérence ?}

Le mot " autonomie » est employé, dans notre société, à propos de réalités très disparates et les conceptions qu'on s'en fait sont souvent fort contrastées. Nous examinerons cette complexité avant de tenter d'en dégager quelques pistes pour une approche sociologique du phénomène.

\section{Des réalités disparates}

On parle d' « autonomie » dans une multitude de domaines différents aujourd'hui. Pour tenter de cerner cette pluralité, nous avons étudié, à l'aide des "alertes » du moteur de recherche Google, tous les articles concernant l'autonomie publiés en langue française sur Internet pendant six mois (d'avril à septembre 2005) ${ }^{33}$ et tous les articles publiés dans Le Monde pendant un peu plus d'un an (d'août 2004 à septembre 2005) dont le titre contenait le mot « autonomie ». Le but n'était pas d'être exhaustif mais de prendre la mesure de la diversité d'emploi du concept. II en ressort que le mot "autonomie » est essentiellement employé dans quatre grands domaines : santé, politique, économie et société.

C'est la santé qui vient en tête en 2004-2005, compte tenu de l'actualité française de l'époque et du projet du gouvernement Raffarin d'instituer une journée de solidarité pour financer la solidarité envers les personnes âgées et handicapées. Les nombreux articles publiés dans ce domaine concernent les personnes dépendantes en général, les handicapés, et enfin - à une seule occasion - l'autonomie des patients emprisonnés.

La matière consacrée au versant politique de l'autonomie est également très abondante. Une partie porte sur le pouvoir étatique et le degré de souveraineté de l'État mais les articles les plus nombreux concernent le pouvoir infra-étatique et l'autonomie gouvernementale. Compte tenu de l'actualité (les élections régionales d'avril 2005), le statut d'autonomie du Pays Basque espagnol est le cas le plus fréquemment traité. Mais beaucoup d'autres sont également évoqués. Plusieurs articles portent sur les perspectives d'autonomie gouvernementale de peuples autochtones, notamment les Indiens du Canada. Enfin, divers mouvements autonomistes sont évoqués. D'autres articles portent sur le pouvoir subétatique (l'autonomie des préfets, des universités, des ports, etc.). Enfin, quelques-uns évoquent des mouvements contestataires ou révolutionnaires, telles les communautés zapatistes du Chiapas.

D'autre part, plusieurs articles portent sur les relations entre individu et société (le besoin d'autonomie des jeunes Chinois, les rapports entre autonomie individuelle et autonomie politique au Québec, etc.). En revanche, rares sont les articles portant sur l'autonomie en éducation, ce qui paraît d'autant plus surprenant que de nombreux ouvrages ont été consacrés à la question.

Enfin, le dernier domaine de publication est l'économie. Des articles portent sur l'autonomie des cadres, l'autonomie des salariés non cadres, le contrôle des salariés

\footnotetext{
${ }^{33}$. En excluant de la recherche l'autonomie des véhicules, des batteries et des ordinateurs...
} 
nomades et l'autonomie de production et de gestion des entreprises. Quelques autres sont consacrés à l'autonomie bancaire et à l'autonomie financière d'un organe de presse (le Corriere della Sera).

Les domaines dans lesquels on emploie le terme " autonomie " aujourd'hui sont donc extrêmement variés. $Y$ a-t-il quelque chose de commun à toutes ces réalités hétéroclites qu'il recouvre?

\section{Des conceptions contrastées}

Pour Berger et Luckmann, " la compréhension sociologique de la "réalité" et de la "connaissance" se situe quelque part à mi-chemin entre celle de l'homme de la rue et celle du philosophe ${ }^{34}$... Examinons donc, successivement, les conceptions que se font de l'autonomie des philosophes, des sociologues et des "hommes de la rue " ou, plus exactement, des non-spécialistes.

C'est de la philosophie que nous vient la notion d'autonomie, et c'est la conception d'un philosophe, Kant, qui a servi de point de départ à notre réflexion. Rappelons, cependant, que l'approche kantienne de l'autonomie est loin d'être partagée de tous les philosophes. D'une part, elle ne correspond qu'à l'une des deux grandes traditions philosophiques relatives à l'autonomie, celle qui prévaut, notamment, en France, tandis qu'en Europe du Nord et dans les pays anglo-saxons, l'autonomie s'inscrit dans une conception individualiste de la liberté ${ }^{35}$, comme sphère d'indépendance négociée, dans laquelle l'État ne doit pas intervenir. D'autre part, si, depuis une cinquantaine d'années, la notion d'autonomie joue un rôle important dans les travaux philosophiques, il n'est pas sûr que les conceptions d'autonomie individuelle ou d'autonomie rationnelle qui prévalent actuellement aient beaucoup à voir avec la conception kantienne ${ }^{36}$.

La notion d'autonomie n'est pas un concept majeur de la pensée sociologique mais est, néanmoins, présente dans la discipline, depuis ses débuts, à propos de diverses questions : l'autonomie de l'acteur par rapport au déterminisme social (Marx et Durkheim étudient les forces sociales, structures et normes qui pèsent sur les individus, tandis que Weber met l'accent sur les marges d'autonomie) ; l'autonomie de l'État et des institutions (Marx et Engels, puis Lénine, présentent essentiellement l'État et les institutions comme des instruments de la classe dominante, tandis que Poulantzas leur reconnaît une autonomie relative); l'autonomie de l'acteur face au contrôle social, qui nous ramène à Durkheim et mérite qu'on s'y arrête un instant. Durkheim, en effet, voit en l'éducation morale la contrainte la plus efficace de la société sur ses membres mais souligne que, loin d'être un dressage, elle doit faire appel à l'autonomie de la volonté. Cette "autonomie " est très différente de l'autonomie kantienne, à laquelle Durkheim reproche d'être trop exclusivement fondée sur l'individu alors que, pour lui au contraire, elle relève d'une exigence d'intériorisation des raisons pour lesquelles l'individu est attaché au groupe ${ }^{37}$. Norbert Élias pousse plus loin l'analyse et fait apparaître combien l'autonomie est un produit d'interaction. Le propre de l'homme, démontre-t-il en effet, est d'être d'une nature intégralement sociale et le comportement autonome est une obligation qui résulte de la mobilité des individus et de la réduction de leur encadrement par la famille, les

\footnotetext{
${ }^{34}$. Berger et Luckmann, 1996, p. 8.

${ }^{35}$. On prendra garde, cependant, de ne pas confondre autonomie et liberté, ainsi que le rappelle Dworkin (1998, p. 14).

${ }^{36}$. Cf. O'Neill, 2004.

${ }^{37}$. Cf. Durkheim, 1974, p. 101.
} 
communautés pré-étatiques et les groupes restreints. Les individus « peuvent bien plus librement décider de leur sort. Mais aussi doivent-ils décider de leur sort. Non seulement ils peuvent devenir plus autonomes, mais ils le doivent. À cet égard, ils n'ont pas le choix ${ }^{38}$. Enfin, depuis des années, Alain Touraine théorise l'autonomie du sujet. Cependant, dans le couple autonomie/sujet, c'est au sujet qu'il accorde toute son attention, c'est lui qu'il définit, régulièrement : "Ce que je nomme sujet », écrit-il, par exemple, en 2005, " est l'affirmation, dont les formes sont changeantes, de la liberté et de la capacité des êtres humains de se créer et de se transformer individuellement et collectivement $"{ }^{39}$ L'autonomie est contenue implicitement dans la définition du sujet que donne Touraine, qui parle, finalement, davantage de "liberté ", voire d' "indépendance " ${ }^{40}$ que d' "autonomie " du sujet, contrairement à ses proches Michel Wieviorka $^{41}$ ou François Dubet ${ }^{42}$.

Nous ne disposons d'aucune enquête sur les représentations sociales de l'autonomie et ne pouvons donc pas évoquer ce qu'elle signifie exactement pour l' " homme de la rue ». Cependant, l'examen des articles précités nous a montré l'extrême diversité d'emploi du mot " autonomie » et suscite plusieurs interrogations. En premier lieu se pose la question de savoir s'il existe quelque chose de commun à toutes les réalités à propos desquelles le mot est employé. L'opinion qui prévaut généralement sur ce sujet est que les différents aspects de l'autonomie n'ont « rien à voir » les uns avec les autres ${ }^{43}$. De fait, il n'y a vraisemblablement rien de commun, en effet - pour reprendre les exemples d'actualité que nous évoquions ci-dessus entre le degré de souveraineté de l'État, l'autonomie des préfets ou des institutions bancaires et l'autonomie que les anarchistes revendiquent... On ne voit pas bien non plus, a priori, ce qu'il pourrait y avoir de commun entre des personnes âgées ou handicapées, des zapatistes, des autonomistes et les rédacteurs d'un quotidien italien. Encore que... N'avons-nous pas affaire, dans tous ces derniers cas, à des êtres humains qui refusent une identité imposée, affirment leur liberté de déterminer le cours de leur vie et éprouvent un désir de reconnaissance? II nous semble que lorsque le mot "autonomie " n'est pas employé métaphoriquement à propos des institutions étatiques, bancaires ou des entreprises, mais à propos des individus et des groupes humains, il désigne un processus de subjectivisation et un questionnement adressé à la société dans son ensemble. En second lieu se pose la question de savoir si une conception sociale de l'autonomie émerge des usages courants que nous avons relevés. Selon nous, on peut apporter une réponse positive à cette question; l'autonomie paraît être perçue comme la combinaison de trois éléments : la faculté de choisir par soi-même (et d'émettre ses propres normes), la capacité d'agir sans l'intervention d'un tiers et le fait - pour un individu ou une collectivité - de disposer des ressources nécessaires à la réflexion et à l'action. Deux autres questions se posent, enfin : d'une part, est-il raisonnable d'employer le même concept d'autonomie aussi bien à propos d'un individu que d'un groupe social ? D'autre part, où commence et où finit l'autonomie ? L'amplitude paraît, en effet, fort large de la dépendance totale à la pleine souveraineté...

\section{Synthèse}

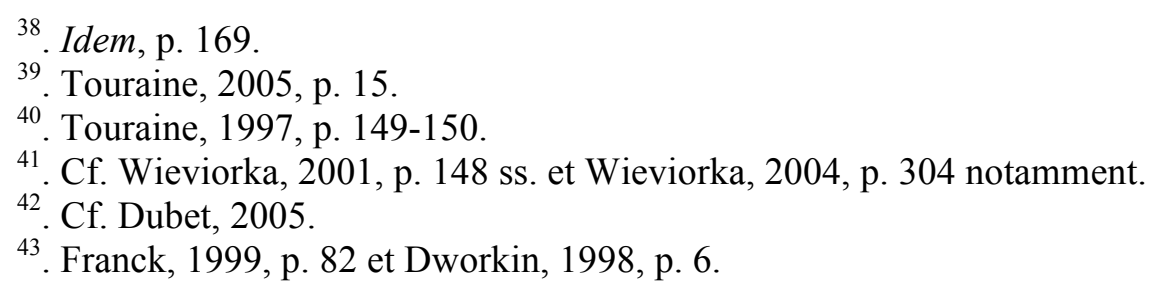


Par-delà leur hétérogénéité, il nous semble qu'il existe un point commun à toutes les approches et conceptions de l'autonomie que nous avons évoquées : la réflexivité sur laquelle elle est fondée. En effet, de l'approche kantienne au sens commun en passant par les travaux de Durkheim, Élias ou Touraine, l'autonomie repose toujours sur la réflexivité. Or, ont montré Giddens ${ }^{44}$ et Beck $^{45}$, la réflexivité sociale constitue l'une des caractéristiques majeures de l'époque de "modernité tardive » en laquelle nous vivons; notre époque est donc bien favorable à l'émergence de l'autonomie. Pour autant, la réflexivité n'en est pas moins limitée. D'une part, remarque avec humour Gerald Dworkin, si l'on attend de la réflexivité qu'elle soit « un processus conscient, complètement articulé et explicite, il apparaîtra que ce sont principalement des professeurs de philosophie qui pratiquent l'autonomie ${ }^{46}$ ! D'autre part - et surtout - la réflexivité se heurte aux limites incompressibles de la capacité des êtres humains à pénétrer jusqu'au tréfonds de leur inconscient et à mettre en question les acquis intimes de leur socialisation. II est donc clair que l'autonomie absolue est inatteignable et qu'il ne peut y avoir qu'une autonomie relative.

Nous avons souligné la multiplicité des usages courants du mot autonomie et malgré leur apparente irréductibilité ainsi que l'avis négatif des spécialistes - avons cru déceler une parenté entre les usages du concept qui concernaient non pas les institutions mais les êtres humains. Posons donc la question en termes plus précis: existe-t-il, ou non, une continuité entre l'autonomie individuelle et les différentes formes d'autonomie collective? Beaucoup d'avis tranchés s'expriment en faveur d'une réponse négative. Ainsi, dans le débat qui, outre-Atlantique, a opposé les libéraux aux communautariens pendant une vingtaine d'années, les libéraux considèrent que les individus ne sont pas définis par leurs appartenances: ils sont libres de remettre en question toute forme de participation à un groupe et, donc, seule l'autonomie individuelle compte. Tandis que pour les communautariens, les individus sont "enchâssés " (embedded) dans un contexte social, ethnique, religieux, etc. ; leurs valeurs sont le produit des valeurs du groupe auxquels ils appartiennent et l'individuation est inséparable de la socialisation. Donc, selon eux, d'une part, le libéralisme nie une dimension essentielle de la personne humaine en ignorant ses appartenances et, d'autre part, l'autonomie individuelle est néfaste car elle favorise l'égoïsme et le déclin des valeurs de la communauté ${ }^{47}$. À nos yeux, les communautariens ont raison d'affirmer que les individus sont "enchâssés " dans des cultures qui doivent être reconnues pour éviter que les minoritaires soient discriminés. C'est un point qui nous paraît fondamental et qui rejoint, d'une certaine façon le propos d'Élias, selon lequel le propre de l'homme est d'être d'une nature intégralement sociale. En revanche, les libéraux ont parfaitement raison de souligner que les individus doivent pouvoir choisir leur appartenance. Nous voyons donc une continuité et non pas une rupture entre les formes individuelle et collective de l'autonomie : pour éviter toute forme de totalitarisme, l'autonomie d'un groupe devrait s'accompagner de la possibilité pour ses membres d'en faire partie ou de le quitter. Par ailleurs, au sein des différentes formes d'autonomie collective, s'il est généralement convenu que l'autonomie révolutionnaire et l'autonomisme n'ont « rien à voir ", il nous semble, en réalité, que, là encore, il peut y avoir continuité. Dans le cas des Indiens du Chiapas, par exemple, n'y a-t-il pas corrélation entre l'ethnicité et

\footnotetext{
44 . Giddens, 1994.

45 . Beck, 2000.

46 . Dworkin, 1998, p. 17.

47 . Etzioni, 1996.
} 
une lutte sociale globale en terme d'autonomie ? En fait, selon nous, l'autonomie ne prend tout son sens qu'en combinant ces trois dimensions: l'individu, la (ou les) communauté(s) choisie(s) (ethnique, de genre ${ }^{48}$, ou autre) et la société globale.

Comment parvenir, dans ces conditions, à une définition opératoire de l'autonomie sans sombrer dans la confusion ? Reprenons, pour ce faire, les éléments que nous avons progressivement accumulés. L'autonomie n'est pas la liberté ; elle est fondée sur la réflexivité ; il n'existe pas d'autonomie absolue, tout est question de degré ; plusieurs types d'autonomie différents peuvent être distingués mais une continuité se dessine entre l'autonomie individuelle et l'autonomie collective ; l'autonomie ne prend tout son sens qu'en combinant les trois dimensions de l'individu, de sa (ses) communauté(s) choisie(s) et de la société ; enfin, l'autonomie collective doit reposer sur la possibilité pour l'individu de quitter ses groupes d'appartenance. Reste à proposer de cette autonomie individuelle une définition peut-être plus terre à terre et moins exigeante que la conception kantienne, qui demande en quelque sorte à l'individu de se comporter en " saint laïque »... II nous semble alors que l'autonomie individuelle pourrait être conçue comme la faculté et la capacité concrète pour les individus d'effectuer les choix et de réaliser les actions qui leur importent, en s'appuyant sur leur auto-réflexion, sans que des formes de manipulation, de tromperie ou de coercition viennent interférer dans leurs choix et leurs actions. Cette définition peut paraître simpliste mais elle nous paraît suffisamment réaliste pour permettre d'étudier sociologiquement l'autonomie, à l'aide de questionnaires, d'entretiens et d'échelles d'attitude ${ }^{49}$, par exemple. L'autonomie, cependant, n'est-elle qu'une réalité relative, ou ne peut-elle pas être quelque chose de plus ? un projet ?

\section{QUEL PROJET ?}

Avant d'évoquer les éventuelles vertus de l'autonomie et les moyens de la développer, examinons-en les limites et les insuffisances.

\section{Limites}

Face à la misère qui règne sur la planète, la question de l'autonomie peut, en premier lieu, paraître dérisoire. Les inégalités, en effet, se développent sans cesse entre pays pauvres et riches ainsi qu'à l'intérieur de ces derniers ; la souffrance, la maladie et la faim se répandent dans de vastes régions du globe : près de huit cents millions d'être humains sont menacés de famine aujourd'hui et la faim est encore la principale cause de mortalité dans le monde, tuant 24000 personnes par jour... Dans ces conditions, disserter d'autonomie peut sembler désinvolte ou absurde. Pourtant, la dimension de l'autonomie n'est pas absente de ces drames: laisser la misère s'aggraver est non seulement inhumain, mais c'est aussi limiter considérablement les conditions élémentaires d'autonomie de millions de personnes. Peut-on, en effet, être autonome lorsqu'on vit dans une intense souffrance ${ }^{50}$ ? D'autre part, la détresse de ces populations peut constituer un terreau favorable à l'apparition de mouvements

48. Voir les travaux de Marilyn Friedman sur l'autonomie, le genre et la politique (2003).

49. Il nous semble, notamment, qu'en s'inspirant de la définition simple que nous donnons de l'autonomie - qui peut évidemment être améliorée - , on pourrait construire des échelles d'autoévaluation de l'autonomie du type des échelles d'auto-positionnement politique.

${ }^{50}$. Les avis sont partagés sur cette question. Pour Joseph Raz, les vies de grande souffrance ne peuvent pas être réellement autonomes (Raz, 1986, p. 373-374) alors que pour Friedman " même les circonstances les plus désespérées et les plus tragiques» peuvent offrir une possibilité de choix autonome. (Friedman, 2003, p. 25. Traduit de l'anglais par nos soins). 
violents qui portent atteinte à l'autonomie d'autrui ; surtout si elles ont le sentiment d'être totalement exclues de la modernité ${ }^{51}$.

La deuxième limite de l'autonomie est qu'elle peut représenter un danger pour la société. En premier lieu, dans l'esprit de nombreux observateurs, parce qu'elle favorise un individualisme débridé, qui menace le bien commun. C'est l'avis, nous l'avons vu, de certains communautariens, tel Amitai Etzioni. C'est aussi l'avis d'Albert O. Hirschman, qui considère que, si l'autonomie individuelle peut être utile à une société à un stade de son développement, elle risque ensuite de devenir problématique $^{52}$. Pourtant, l'autonomie telle que nous l'avons définie ne conduit pas nécessairement à l'éclatement de la société : elle est simplement la faculté et la capacité concrète pour les individus d'effectuer les choix et de réaliser les actions qui leur importent, hors de toute hétéronomie. Cependant, elle peut également être dangereuse à un autre égard: certaines personnes peuvent choisir, hors de toute contrainte, de se comporter d'une façon qui leur est (ou paraît leur être) néfaste. Par exemple, elles peuvent choisir délibérément de se priver de liberté. L'autonomie doitelle avoir un fondement substantiel - les choix effectués de façon autonome doiventils être conformes à certaines valeurs (et notamment à l'autonomie elle-même) ? ou, au contraire, doit-elle être neutre? L'avis de Marilyn Friedman, philosophe et militante féministe, est que l'autonomie doit être neutre, même si elle nuit à son bénéficiaire. Tel est également l'avis de Gerald Dworkin, qui cite l'exemple d'Ulysse, se privant délibérément de liberté pour écouter le chant des sirènes ${ }^{53}$. On ne peut nier, toutefois, les risques que comporte une autonomie neutre.

L'autonomie, enfin, n'est nullement la panacée. Rien ne garantit, en premier lieu - si l'on s'en tient à une définition non kantienne de l'autonomie, comme celle que nous avons proposée - , qu'elle n'entre pas en contradiction avec de plus hautes valeurs morales. Par exemple, un individu peut ne réfléchir à ses choix et à ses actions qu'à l'aune de ses désirs et ignorer les conséquences de ses actes sur autrui. Ce serait faire preuve d'égoïsme; mais serait-ce l'autonomie qui serait à blâmer ou l'usage que cet individu en fait ? Rien dans l'autonomie ne suppose, en effet, une indifférence égoïste à l'autre ${ }^{54}$. En second lieu, l'autonomie ne peut fonctionner qu'en certaines circonstances. Les personnes concernées doivent avoir la capacité intellectuelle d'être autonomes; il faut que des alternatives significatives leur soient offertes ; qu'elles puissent considérer ces alternatives en fonction de leurs souhaits et valeurs, sans être manipulées; enfin, les groupes auxquels elles appartiennent doivent pouvoir jouir de l'autonomie en tant que collectivités... En troisième lieu, l'autonomie ne peut être, nous l'avons dit précédemment, que relative. En quatrième lieu, elle ne peut pas - par définition - être imposée, ce qui signifie qu'elle ne peut pas, non plus, être généralisée. Enfin, si l'on en croit certains auteurs, elle risque de n'être accessible qu'aux élites ${ }^{55}$. Les limites de l'autonomie étant posées, examinons à présent ce que pourraient être ses vertus.

\section{Vertus}

51. Cf. Maalouf, 1998 et Wieviorka, 2004.

52. Hirschman, 1995, p. 52.

53 . Dworkin, 1998, p. 14-15.

54 . Cornelius Castoriadis pense même que «l'on ne peut vouloir l'autonomie sans la vouloir pour tous » $(1999$, p. 159).

55. Cf. Castells, 1998, vol. II, p. 22. Ce point est néamoins contesté : cf., notamment, Friedman, 2003, p. 46. 
Nous envisagerons les qualités de l'autonomie sous deux angles complémentaires ; l'un, "réactif »: les réponses à des problèmes contemporains ; l'autre, "projectif »: les visées de transformation de la société. En premier lieu, l'autonomie peut constituer l'une des réponses à la crise de la représentation que la plupart des pays développés traversent ${ }^{56}$, en donnant aux citoyens un rôle d'acteurs sur les grandes questions qui les préoccupent. Par exemple, dans le cas français, Jean-Jack Queyranne, président du conseil régional Rhônes-Alpes, suggère que « réformer l'État, c'est accorder l'autonomie régionale »; selon lui, en effet, " face à la crise de l'État et de la démocratie représentative, la régionalisation est une alternative. [...] Elle représente la forme moderne de la citoyenneté ${ }^{57}$. " Sur un plan plus large, l'autonomie peut constituer une réponse à différentes formes d'agressivité nationaliste. D'une part, dans sa forme collective (l'autonomie de groupe), elle peut fournir une réponse souple au « national-étatisme » (ou nationalisme central) - qui a tendance à nier les droits des minorités -, en détendant les relations entre le centre et la périphérie ${ }^{58}$. Dans sa forme individuelle, en revanche, elle peut préserver les individus du "national-communautarisme » (ou nationalisme ethnique), en leur permettant de choisir librement de s'identifier ou non aux groupes et communautés qui pourraient vouloir exercer une emprise sur eux. Enfin, l'autonomie peut même, à plus ou moins long terme, être l'une des façons de résoudre les difficultés que la globalisation engendre. Selon Ulrich Beck, en effet, il n'existe pas de réponse à la globalisation au niveau de l'État-nation et il faut donc faire preuve d'imagination pour trouver une solution alternative ${ }^{59}$. Lui-même, en l'absence d'un État mondial, plaide en faveur de l'avènement d'États transnationaux ${ }^{60}$, pense qu'il faut s'appuyer sur les particularités régionales ${ }^{61}$ et affirme que le développement de l'autonomie personnelle est inévitable ${ }^{62}$. Mais nous abordons là l'autre versant de la question: l'autonomie comme projet de transformation de la société.

En second lieu, l'autonomie, exprimant la capacité des êtres humains à raisonner en conscience, à faire preuve de réflexivité et à s'autodéterminer, peut constituer la base d'une nouvelle étape démocratique. Tel est l'avis de David Held, qui consacre l'avant-dernier chapitre de son ouvrage Models of Democracy à l'autonomie démocratique, et définit ainsi le principe d'autonomie ${ }^{63}$ qui devrait, selon lui, être considéré comme le fondement de la démocratie ${ }^{64}$. Cornelius Castoriadis va beaucoup plus loin et fait de l'autonomie le cœur d'un projet révolutionnaire qui remet totalement en cause le pouvoir et vise à « l'auto-institution explicite de la société par l'activité collective, lucide et démocratique ॥ 65 . Le projet d'autonomie tel qu'il le conçoit implique de façon circulaire les individus, la société et la culture ; c'est un processus de construction culturel fondé sur la paideia, c'est-à-dire sur l'éducation et la socialisation $^{66}$. Il en pose les conditions dans le texte ci-dessous :

${ }^{56}$. Pharr and Putnam, 2000.

${ }^{57}$. Queyranne, 2005.

${ }^{58}$. Si, toutefois, elle est introduite avec suffisamment de doigté et de finesse (cf. Lapidoth, 1999); sans quoi, elle peut, au contraire, constituer une menace pour l'unité sociale.

${ }^{59}$. Beck, 2000, p. 108.

${ }^{60}$. Beck, 2000, p. 108 ss.

${ }^{61}$. Idem, p. 144 ss.

${ }^{62}$. Idem, p. 147.

${ }^{63}$. Held, 2000.

${ }^{64}$. Held, 2000, p. 303.

65. «L'idée de révolution » in Castoriadis, 1979, p. 331.

${ }^{66}$. Cf. David, 2000, p. 77 ss. 
«Un véritable devenir public de la sphère publique/publique ${ }^{67}$, une réappropriation du pouvoir par la collectivité, l'abolition de la division du travail politique, la circulation sans entraves de l'information politiquement pertinente, l'abolition de la bureaucratie, la décentralisation la plus extrême des décisions, le principe: pas d'exécution des décisions sans participation à la prise des décisions, la souveraineté des consommateurs, l'auto-gouvernement des producteurs - accompagnés d'une participation universelle aux décisions engageant la collectivité, et d'une auto-limitation. " 68

De l'autonomie personnelle à l'autonomie révolutionnaire de Castoriadis en passant par l'autonomie territoriale ou régionale et l'autonomie démocratique de David Held, on voit combien le projet d'autonomie peut être protéiforme. S'agit-il alors d'un fourre-tout incohérent? Le lecteur aura compris que nous ne le pensons pas. À nos yeux, ce qui distingue les différentes formes d'autonomie que nous venons d'évoquer entre elles, c'est, d'une part, l'angle selon lequel on les approche (l'individu, la/les communauté(s) choisie(s), la société), d'autre part, le filtre disciplinaire auquel on recourt (philosophie, sociologie, science politique...) et, enfin, le degré d'autonomie que l'on considère (de la simple réforme à la perspective révolutionnaire). Donc, selon nous, les fins étant apparentées, les moyens le seront également.

\section{Moyens}

Nous distinguerons trois voies d'accès à l'autonomie individuelle ou collective, qui nous semblent toutes essentielles: la reconnaissance de l'Autre ; l'éducation; et le fait de fonder le droit sur l'individu.

La reconnaissance de l'Autre constitue l'un des piliers du projet d'autonomie, à l'échelle de l'individu, de ses communautés d'appartenance, comme de la société globale. Comment, en premier lieu, l'individu peut-il se libérer de l'hétéronomie ? En tentant d'éliminer le discours de l'Autre ${ }^{69}$ qu'il découvre en lui ? C'est impossible, puisque nous sommes tous constitués de l'Autre. La solution est donc de reconnaître l'Autre en soi et d'instaurer « un autre rapport entre le discours de l'Autre et le discours du sujet ${ }^{70}$. En second lieu, concernant les communautés d'élection, prenons l'exemple de l'ethnicité, où la question de l'autonomie se pose le plus instamment $^{71}$. La principale condition de réussite de l'autonomie territoriale, nous explique Ruth Lapidoth, « réside dans une atmosphère générale de conciliation et de bonne volonté ${ }^{72}$. Celle-ci repose, à la fois, sur la reconnaissance de la minorité par l'État central et le groupe majoritaire, et sur la reconnaissance de ces derniers par la minorité qui bénéficie de l'autonomie. En outre, il faut également que la minorité autonome reconnaisse ses propres minorités, ce qui n'est pas toujours le cas. Enfin,

${ }^{67}$. Castoriadis distingue «trois sphères où se jouent les rapports des individus et de la collectivité entre eux et avec leur institution politique : la sphère privée — oikos —, la sphère publique/privée agora -, la sphère publique/publique, que dans le cas d'une société démocratique [il appelle] ecclésia ». (1997, p. 62-72). La sphère publique/publique est «l'instance où sont discutées et décidées les œuvres et les entreprises qui concernent et engagent la collectivité entière » (Ibid).

${ }^{68}$. Castoriadis, 1997, p. 74.

69. Qu'il s'agisse - mais tout est lié — de l'inconscient, des influences familiales, des socialisations successives, etc.

${ }^{70}$ Castoriadis, 1999, p. 155.

${ }^{71}$. Mais on pourrait aussi bien choisir toute autre forme de communauté (de genre, de sexualité, de handicap, etc.)

\footnotetext{
${ }^{72}$. Lapidoth, 1999, p. 32.
} 
en troisième lieu, si l'on conçoit l'autonomie comme un projet global de transformation de la société, la question de la reconnaissance - "besoin humain vital " selon Charles Taylor $^{73}$ — se pose à l'échelle de la planète. Tous les êtres humains ont besoin de reconnaissance en matière d'amour, de droit et de solidarité, explique Axel Honneth, sous peine de souffrance sociale; la non-reconnaissance dans l'un quelconque de ces trois domaines est une forme de mépris, qui affecte gravement l'identité ${ }^{4} \ldots$

Reconnaître l'Autre, cependant, n'est pas chose aisée, qu'il s'agisse de le reconnaître en soi ou hors de soi, comme sujet digne d'amour, de droits et de solidarité - au même titre que soi... Cela suppose une grande maturité dont l'éducation est la clef. Seule une éducation appropriée peut, en effet, permettre à l'individu de s'autonomiser par rapport à son inconscient ou aux influences et déterminismes qui pèsent sur lui, permettre aux minorités et aux majorités de se respecter mutuellement, et aux habitants de la planète de faire preuve d'une véritable solidarité les uns envers les autres. C'est ce que souligne Castoriadis, pour qui la paideia est l'institution fondamentale de l'autonomie, chargée d'effectuer un "dressage des individus vers/pour la communauté » afin d'amener "le petit monstre nouveau-né », ce « faisceau de pulsions et d'imagination » à l'état d'être humain ${ }^{75}$.

L'autonomie, enfin, peut être fondée sur le droit, mais un droit dont la source serait l'individu lui-même. II s'agit de protéger les individus des pouvoirs, d'où qu'ils viennent (des États, des communautés, des firmes transnationales...) et d' " incarner dans les institutions, autant que faire se peut, l'autonomie individuelle et sociale ${ }^{76}$. L'un des fondements théoriques de ce droit pourrait être la liberté culturelle, "la faculté donnée aux individus de vivre et d'être ce qu'ils choisissent, en ayant réellement la possibilité de considérer d'autres options ${ }^{77}$. Concrètement, ceci existe peut-être déjà quelque peu, sous la forme de l'autonomie personnelle qui, explique Ruth Lapidoth, « s'applique à tous les membres d'un certain groupe dans un État ou une région déterminée, quel que soit leur lieu de résidence ${ }^{78}$. Toutefois, il est possible d'aller plus loin. Stéphane Pierré-Caps, reprenant les idées de Karl Renner, suggère de séparer l'État de la nation - comme on a jadis séparé l'Église et l'État — et de faire du droit à l'autodétermination un droit individuel: avec la multination et l'autodétermination individuelle, " la volonté de l'individu ainsi exprimée est source de droit, il ne se trouve pas enfermé dans un cadre national préétabli, qui façonne et organise son allégeance à l'État ${ }^{79}$. Thomas M. Franck pense qu'il faut se diriger vers une société civile fondée sur « un individualisme qui donne à chaque personne le pouvoir de définir son identité unique, protégée par un régime de lois nationales et internationales ${ }^{80}$ et vers " une forme de participation directe des personnes à un processus de démocratie représentative global ${ }^{81}$. Enfin, Castoriadis suggère que

${ }^{73}$. Taylor, 1994, p. 42.

${ }^{74}$. Honneth, 2000, p. 163 à 165.

${ }^{75}$. Cornelius Castoriadis, Réflexions sur le «développement» et la «rationalité», in Les Carrefours du Labyrinthe II, Paris, Seuil, 1999, cité par David, 2000, p. $81 \mathrm{n}$.

${ }^{76}$ Castoriadis, L'écrivain et la démocratie [1991], in David, 2000, p. 165.

${ }^{77}$. PNUD, 2004, p. 4.

${ }^{78}$. Lapidoth, 1999, p. 27-28.

${ }^{79}$. Pierré-Caps, 1995, p. 46.

${ }^{80}$. Franck, 1999, p. 44.

${ }^{81}$. Franck, 1999, p. 37. 
l'objectif de l'autonomie serait de faire de chaque individu un "être capable de gouverner et d'être gouverné ${ }^{82}$.

\section{CONCLUSION}

La fréquence avec laquelle le mot «autonomie » est utilisé aujourd'hui pour décrire et analyser les comportements de nos contemporains est sans doute excessive. S'il est vrai que l'hétéronomie autoritaire régresse dans notre société et que la désirabilité de l'autonomie augmente, l'hétéronomie en général ne disparaît pas, la notion d' "autonomie imposée " relève de l'abus de langage et enfin l'autonomie n'apparaît pas automatiquement là où l'hétéronomie reflue. Les domaines dans lesquels le terme " autonomie » est d'usage courant sont multiples et les acceptions philosophiques et sociologiques du concept sont diverses ; on pourrait en conclure qu'il faut procéder à des ruptures et qu'il convient, en particulier, de séparer radicalement ce qui relève de l'individu, du groupe et de la société. Nous ne le pensons pas et considérons, au contraire, que, pour prendre tout son sens en tant que projet de société, l'autonomie doit être conçue en combinant ces trois dimensions. Il faut, néanmoins, se garder de trois écueils : une approche universaliste abstraite de l'autonomie, qui en fait un idéal inaccessible aux humains ; une approche restrictive, qui la réduit à l'accommodement technique de la diversité ethnique; et une approche systématique qui, en voyant de l'autonomie partout, finit par la dissoudre dans l'inconsistance. Selon nous, pour éviter toute forme de totalitarisme, l'individu doit être le fondement de l'autonomie, ce qui nous ramène à l'universalisme mais dans une perspective concrète. La dimension collective de cet individu doit être prise en compte et pleinement reconnue - notamment son appartenance à des groupes ou des communautés - , mais seulement dans la mesure où il le souhaite. Enfin, la question de l'autonomie doit être posée à l'échelle de la société globale et de la solidarité de ses membres. Alors l'autonomie peut devenir non seulement un concept fécond mais un véritable outil au service de la croissance de la démocratie et du développement humain.

\section{BIBLIOGRAPHIE}

Beck Ulrich, What is Globalization ? Cambridge, Polity Press, 2000. - Bell Daniel, The World and the United States in 2013, Daedalus, 1987, vol. 116, n ${ }^{\circ} 3$, p. 1-32. Berger Peter \& Luckmann Thomas, La Construction sociale de la réalité, Paris, Méridiens Klincksieck, 1996. - Besnard, Philippe, L'anomie, Paris, PUF, 1987. Boltanski Luc \& Chiapello Eve, Le Nouvel Esprit du capitalisme, Paris, Gallimard, 1999. - Bouvier Pierre, Le lien social, Paris, Gallimard, 2005. - Castells Manuel, L'ère de l'information, vol. I, II et III, Paris, Fayard, 1998. - Castoriadis Cornelius, Le contenu du socialisme, Paris, UGE «10/18 », 1979. - Castoriadis Cornelius, Les carrefours du labyrinthe, tome v : Fait et à faire, Paris, Seuil, 1997. - Castoriadis Cornelius, L'institution imaginaire de la société, Paris, Seuil, 1999 [1 ${ }^{\text {re }}$ éd. 1975]. Castoriadis Cornelius, De l'autonomie en politique: l'individu privatisé, Le Monde diplomatique, 2000. - Daoust Valérie, De la sexualité en démocratie, Paris, PUF, 2005. - David Gérard, Cornelius Castoriadis. Le projet d'autonomie, Paris, Michalon, 2000. - Davie Grace, Religion in Britain since 1945 : Believing without

${ }^{82}$. Castoriadis, Psychanalyse et politique, in Le Monde morcelé. Les carrefours du labyrinthe III, p. 146, cité in David, 2000, p. 120. 
Belonging, Oxford, Blackwell, 1994. - Dubet François, Pour une conception dialogique de l'individu. L'individu comme machine à poser et à résoudre des problèmes sociologiques, espacestemps.net/document1438.html, 2005. - Durkheim Émile, L'Éducation morale, Paris, PUF, 1974 [1 $1^{\text {re }}$ éd. 1925]. - Durkheim Émile, Le Suicide, Paris, PUF, 1986 [1 $1^{\mathrm{re}}$ éd. 1897]. - Dworkin Gerald, The Theory and Practice of Autonomy, New York-Cambridge, Cambridge University Press, 1998. Ehrenberg Alain, L'individu incertain, Paris, Calmann-Lévy, 1995. - Ehrenberg Alain, La fatigue d'être soi. Dépression et société, Paris, Odile Jacob, 2000. - Élias Norbert, La société des individus, Paris, Fayard, 1991. - Etzioni Amitai, The New Golden Rule: Community and Morality in a Democratic Society, New York, Basic Books, 1996. - Evaraere Christophe, L'autonomie dans le travail : portée et limites, Revue française de gestion, 2001, $\mathrm{n}^{\circ} 134$, juin-juillet-août. — Filliozat Isabelle, L'intelligence du cœur: Confiance en soi, créativité, aisance relationnelle, autonomie, Paris, Marabout, 1998. - Franck Thomas M., The Empowered Self. Law and Society in the Age of Individualism, Oxford, Oxford University Press, 1999. - Freire Paulo, Pédagogie de l'autonomie : savoirs nécessaires à la pratique éducative, Paris, Erès, 2004. - Friedman Marilyn, Autonomy, gender, politics, Oxford, Oxford University Press, 2003. - Gauchet Marcel, Le désenchantement du monde. Une histoire politique de la religion, Paris, Gallimard, 1985. - Gauchet Marcel, La religion dans la démocratie. Parcours de la laïcité, Paris, Gallimard, 1998. - Giddens Anthony, Les conséquences de la modernité, Paris, L'Harmattan, 1994. - Hannum Hurst, Autonomy, Sovereignty, and Self-Determination. The Accomodation of Conflicting Rights [Revised ed.], Philadelphia, University of Philadelphia Press, 1996. - Held David, Models of Democracy [2nd ed.], Cambridge, Polity Press, 2000. Hirschman Albert O., A Propensity to Self-Subversion, Cambridge, Harvard University Press, 1995. - Honneth Axel, La lutte pour la reconnaissance, Paris, Cerf, 2000. Kant Immanuel, Fondements de la métaphysique des mœurs, Paris, Delagrave, 2000. - Kapferer Jean-Noël, L'enfant et la publicité : les chemins de la séduction, Paris, Dunod, 1985. - Lapidoth Ruth, Autonomy. Flexible solutions to ethnic conflicts, Washington D.C., United States Institute of Peace Press, 1997. - Lapidoth Ruth, Régions insulaires et construction européenne : comparaison entre la Corse et les îles Åland, in ECMI (Centre Européen des Questions de Minorités) Autonomies insulaires : Vers une politique de la différence pour la Corse ?, Éd. Albiana, 1999. - Lapidoth Ruth, Autonomie, unité et démocratie, in Le Coadic, 2003. - Le Coadic Ronan (dir.), Identités et démocratie, Rennes, Presses Universitaires de Rennes, 2003. Lipovetsky Gilles, L'ère du vide, Paris, Gallimard, 1983. - Maalouf Amin, Les Identités meurtrières, Paris, Grasset, 1998. - O'Neill Onora, " Autonomie : le roi est nu », Raison publique, $\mathrm{n}^{\circ} 2$, avril 2004. - OMS, Rapport sur la santé dans le monde 2001. La santé mentale: nouvelle conception, nouveaux espoirs, Genève, Organisation mondiale de la Santé, 2001. - Ostwald Martin, Autonomia : its Genesis and Early History, American Philological Association, American Classical Studies, 11, Chico CA, Scholars Press, 1982. - Pharr Susan J., \& Putnam Robert D., Disaffected Democracies. What's troubling the trilateral countries?, Princeton, Princeton University Press, 2000. - Pierré-Caps Stéphane, La multination. L'avenir des minorités en Europe centrale et orientale, Paris, Odile Jacob, 1995. - PNUD, Rapport mondial sur le développement humain - 2004, Paris, Économica, 2004.

Queyranne Jean-Jack, Réformer l'État, c'est accorder l'autonomie régionale, Le Monde, 6 septembre 2005, p. 17. Raz Joseph, The Morality of Freedom, Oxford, Clarendon Press, 1986. - Taylor Charles, Multiculturalisme. Différence et 
démocratie, Paris, Flammarion, 1994. - Touraine Alain, Pourrons-nous vivre ensemble ? Égaux et différents, Paris, Fayard-Le Livre de Poche, 1997. - Touraine Alain, Un nouveau paradigme: pour comprendre le monde d'aujourd'hui, Paris, Fayard, 2005. - Touraine Alain, Dubet François, Hegedus Zsuzsa, Wieviorka Michel, Le pays contre l'État, Paris, Seuil, 1981. - Wieviorka Michel, La différence, Paris, Balland, 2001. - Wieviorka Michel, La violence, Paris, Balland, 2004. 